\title{
ctDNA Clearance and Radiographic Resolution of Disease in Response to Dual Checkpoint Inhibition in Metastatic Microsatellite Stable Colorectal Cancer with a High Tumor Mutation Burden
}

\author{
Charles J. Schneider ${ }^{a} \quad$ Michael Krainock ${ }^{b}$ Allyson Koyen Malashevich ${ }^{b}$ \\ Meenakshi Malhotrab $^{b}$ Perry Olshan $^{b}$ Paul R. Billings ${ }^{b} \quad$ Alexey Aleshin $^{b}$ \\ ${ }^{a}$ Abramson Cancer Center at the University of Pennsylvania, Philadelphia, PA, USA; ${ }^{b}$ Natera, \\ Inc., San Carlos, CA, USA
}

\author{
Keywords \\ Metastatic colorectal cancer · Circulating tumor DNA · Carcinoembryonic antigen · \\ Noninvasive biomarkers · Microsatellite instability high $\cdot$ Immunotherapy
}

\begin{abstract}
Immunotherapy (IO) has increasingly been demonstrated to provide therapeutic benefit to patients with metastatic colorectal cancer $(\mathrm{mCRC})$. However, only a subset of $\mathrm{mCRC}$ tumors respond to IO. Monitoring response with tumor biomarkers like carcinoembryonic antigen (CEA) has been challenging in patients with microsatellite stable (MSS) mCRC due to low expression of CEA (CEA/lo). Noninvasive blood-based biomarkers such as circulating tumor DNA (ctDNA) can inform early treatment response and augment radiographic monitoring. We describe a case study of a patient with chemotherapy-refractory CEA/lo MSS MCRC, with metastatic disease present in a cardiophrenic lymph node. The patient was given 2 cycles of combination IO (ipilimumab/nivolumab). Response was monitored by ctDNA using a multiplex PCR nextgeneration sequencing assay, CEA, and CT scan. After IO administration, ctDNA levels rapidly declined, becoming undetectable. This was concurrent with radiographic resolution of the lymph node metastasis. Serial monitoring of CEA during this same period was uninformative, with no significant changes observed. Significant decline in ctDNA identified metastatic response to $\mathrm{IO}$ in a patient with CEA/lo, MSS MCRC and was concurrently validated by CT scan. This case study provides evidence that ctDNA can be used as a prospective surrogate for radiographic tumor response.
\end{abstract}




\section{Background}

High microsatellite instability (MSI-H) metastatic colorectal cancer (mCRC) is associated with a beneficial response to immunotherapy (IO), with durable responses observed in patients treated with nivolumab and ipilimumab [1]. Additionally, tumor mutational burden (TMB) may be independently predictive of IO responsiveness in these tumors [1-3]. Less is known about IO treatment responsiveness in patients with microsatellite stable (MSS) mCRC with intermediate-to-high mutational burden. Previous studies have indicated the unreliability of conventional tumor markers such as carcinoembryonic antigen (CEA) in accurately determining the risk of recurrence in these tumors [4]. Also, in patients with tumors that do not express significant quantities of CEA (the CEA/lo phenotype), this tumor marker is not useful in monitoring $\mathrm{mCRC}$ disease status [5]. Thus, alternative serologic surrogates that could more accurately reflect systemic disease burden, such as circulating tumor DNA (ctDNA), are being investigated in MSS mCRC patients [6].

We postulated that correlation of ctDNA with radiographic findings could help establish ctDNA as a useful adjunct to the assessment of tumor status, including disease responsiveness to systemic therapy. We describe a particularly illustrative case of a patient with stage IV MSS CEA/lo mCRC, harboring a PALB2 germline mutation, with a TMB of 15.4 mutations per megabase and a PD-L1 CPS score of 26. The patient responded rapidly and completely to combination IO as assessed by both radiographic imaging and blood-based ctDNA testing.

\section{Case Study}

In November 2017, a 55-year-old female with a PALB2 germline mutation presented with a right-sided colonic adenocarcinoma, with involvement of the omentum and liver. The patient was observed to have a slightly elevated CEA level $(7.6 \mathrm{ng} / \mathrm{mL})$ in December 2017. In general, the patient's CEA remained in the normal range post-presentation, peaking at $3.5 \mathrm{ng} / \mathrm{mL}$, indicating that the tumor is a low CEA producer (CEA/lo) (Fig. 1). The patient received 6 cycles of neoadjuvant FOLFOX, followed by an extended right hemicolectomy, omentectomy, and partial liver resection, which was performed in May 2018 (Fig. 1). The resected tissues revealed a moderately differentiated adenocarcinoma in the cecum, demonstrating a poor response to c-hemotherapy, 0/23 disease-positive lymph nodes, one focus of adenocarcinoma in the liver with clear margins, and focal omental involvement resected with clear margins. The patient was deemed no evidence of disease after surgery.

The patient subsequently underwent 6 cycles of adjuvant FOLFOX. Oxaliplatin was omitted after 3 cycles secondary to peripheral neuropathy (Fig. 1a). Soon after, the patient experienced an FDG-avid peritoneal recurrence on PET/CT between the anterior abdominal wall and stomach. Recurrence occurred approximately 620 days after surgery (Fig. 1,2). The metastatic focus was subsequently resected with negative margins, with tumor profile demonstrating a TMB of 15.4 mutations per megabase, mismatch repair proficiency (pMMR), a PD-L1 CPS score of 26, and a MSS phenotype.

Initial ctDNA analysis was performed using a multiplex PCR next-generation sequencing assay (Signatera ${ }^{\mathrm{TM}}$ ) designed from the resected peritoneal metastasis. The patient was found to be ctDNA positive with 1.83 mean tumor molecules per $\mathrm{mL}$ of plasma (MTM/mL) (Fig. 1b). Following peritoneal metastasectomy, the patient was noted to have a cardiophrenic lymph node enlarged from 5 to $12 \mathrm{~mm}$, concerning for metastasis. Blood-based ctDNA levels correspondingly doubled during this period. 


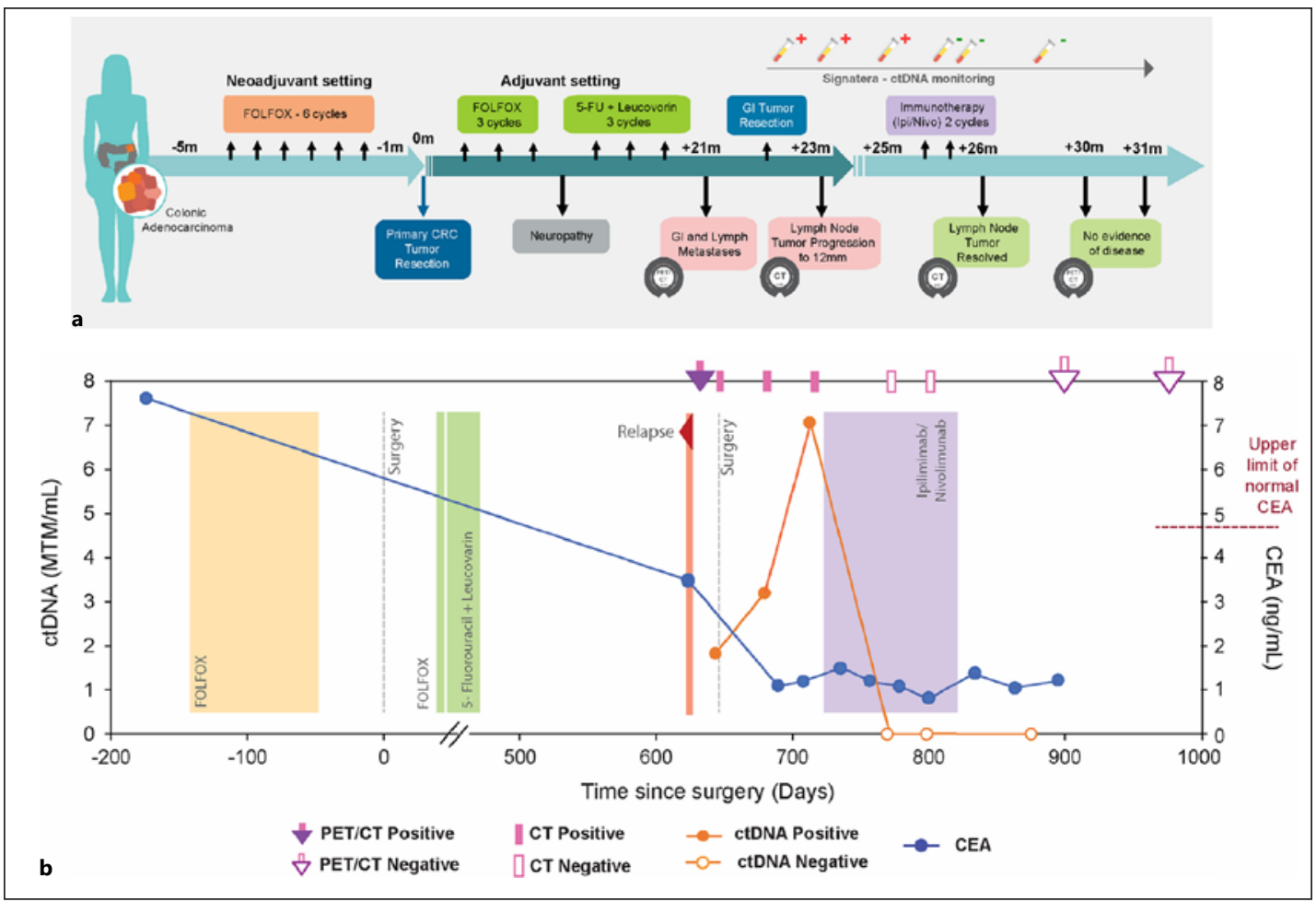

Fig. 1. Clinical course and ctDNA and CEA monitoring. a Patient plot detailing the timeline of treatments administered, PET/CT scanning, and ctDNA monitoring. $\mathbf{b}$ ctDNA and CEA levels over time, measured in number of days since surgical resection of the primary tumor. PET and CT scans, relapse, surgery, and therapeutic treatment windows are represented. ctDNA, circulating tumor DNA; CEA, carcinoembryonic antigen.

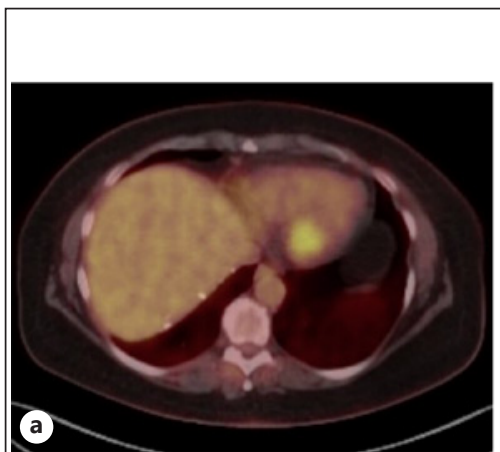

PET/CT Scan $~ 640$ days post-surgery

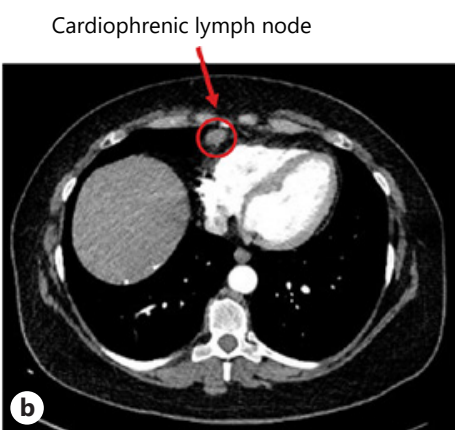

CT Scan 690 days post-surgery
Cardiophrenic lymph node - Resolved

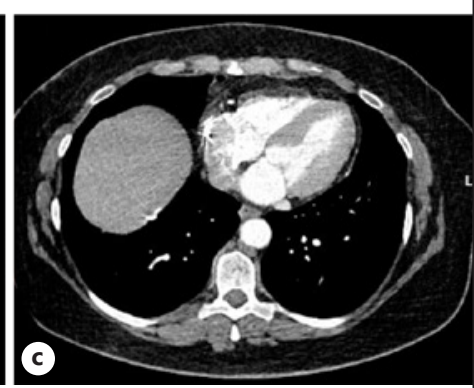

CT Scan $~ 780$ days post-surgery

Fig. 2. PET/CT scan results. a, b The presurgery PET/CT (640 days after initial surgery) showed an FDGavid cardiophrenic lymph node measuring $5 \mathrm{~mm}$ (in addition to the peritoneal/perigastric mass) that was determined in retrospect to represent a second site of disease recurrence. c Following 2 of 4 planned cycles of combination IO, the enlarged cardiophrenic lymph resolved radiographically with "no enlarged thoracic lymph nodes and no evidence of intrathoracic metastatic disease" reported on CT chest (approximately 780 days after initial surgery). IO, immunotherapy.

Given the TMB score of 15.4, the presence of a PALB2 germline mutation, and the elevated PD-L1 score of 26, the patient was planned for 4 cycles of $3 \mathrm{mg} / \mathrm{kg}$ nivolumab and $1 \mathrm{mg} / \mathrm{kg}$ ipilimumab. Following 2 cycles of combination IO, the patient's ctDNA became undetectable 
(Fig. 1) concurrent with radiographic resolution of the cardiophrenic lymph node (Fig. 2). To date, the patient remains in remission.

\section{Discussion}

Here, we present a case of rapid ctDNA clearance correlating with radiographic resolution of metastatic disease after 2 cycles of combination IO in a patient with MSS mCRC. The tumor tested positive for a mutation in PALB2, with a TMB of 15.4 mutations per megabase and a PD-L1 score of 26.

TMB is a controversial tumor-specific parameter that can potentially predict CRC responsiveness to IO, independent of MSI-H status. A recent study by Schrock et al. [1] examined a cohort of 22 patients with MSI-H mCRC treated with PD-1 or PD-L1 inhibitors. This study showed that among tested variables, TMB was most strongly associated with objective response and progression-free survival $(p<0.001)$ [1]. Although a high TMB is usually correlated with microsatellite instability, 1 tissue exploration study of MSS CRC tumors showed that 164 of 5,702 (2.9\%) cases demonstrated a high TMB (range: 11.7-707.2 mut/Mb) [2]. This suggests that a subset of MSS tumors (MSS/TMB-high) may respond to IO. In a randomized phase 2 study of Canadian patients with chemotherapy-refractory CRC (C0.26), patients were randomized to receive either tremelimumab plus durvalumab every 28 days or best supportive care. This study further correlated MSI and TMB status with patient outcome. Interestingly, they found that MSS TMB-high ( $\geq 28$ variants per megabase) patients had the greatest OS benefit (HR $=0.34 ; 90 \%$ CI: $0.18-0.63 \% ; p=0.004$ ) of receiving IO, suggesting that high TMB may be a crucial predictor of disease-associated benefit to I0 treatment [7].

Of note, our patient harbors a germline mutation in $P A L B 2$, a DDR pathway gene. A recent study found that alterations in the DDR were associated with a high TMB in $21 \%$ of advanced colorectal, gastroesophageal, or small bowel carcinomas, across 17,486 patients [8]. The extent to which DDR pathway alterations and high TMB factored into patient responses to IO was unclear. The described patient also had a high PD-L1 CPS score, which has been studied extensively in other malignancies as a determinant of disease responsiveness to IO. PD-L1 CPS scoring has yet to be studied in mCRC.

Serial ctDNA monitoring has been studied by Parikh et al. [9] to provide early indication of response to systemic chemotherapy in patients with metastatic gastrointestinal cancer. Out of 138 patients enrolled, 101 were evaluable for ctDNA and 68 for tumor markers, which were measured at scheduled intervals during therapy. The 4-week change in ctDNA levels more effectively predicted clinical benefit than tumor markers, as measured by radiographic responses, with a sensitivity of $60 \%$ versus $24 \%$ ( $p=0.0109)$ [9]. Serial ctDNA has also been evaluated as a tool in assessing tumor response to IO in patients with non-small cell lung cancer, uveal melanoma, and MSI-H mCRC, treated with either nivolumab or pembrolizumab [10]. Patient ctDNA status at week 8 was a significant prognostic factor for PFS and OS (HR $=10.2$ and 15; 95\% CI: 2.5-41\% and 2.5-94.9\%; $p<0.001$ and 0.004, respectively), leading to our decision to use 8 weeks as our first ctDNA detection time point.

\section{Conclusion}

We present a single-case validation using high TMB, independent of MSI or MMR status, in predicting $\mathrm{mCRC}$ responsiveness to IO. We identified that ctDNA was a more useful indicator of disease status than CEA production levels, in a CEA/lo phenotype tumor. Our case study supports ctDNA as a prospective surrogate for radiographic tumor response, which

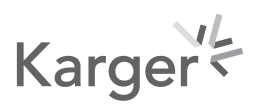


could lead to routine, noninvasive, and cost-effective patient follow-up during a course of systemic treatment.

\section{Statement of Ethics}

This study was conducted ethically in accordance with the World Medical Association Declaration of Helsinki. Written informed consent was obtained from the patient for publication of this case report and any accompanying images.

\section{Conflict of Interest Statement}

C.J.S. has nothing to disclose. M.K., A.K.M., M.M., P.O., P.R.B., and A.A. are employees of Natera, Inc., with stock/options to own stock in the company.

\section{Funding Sources}

This study was supported by Natera, Inc. This study received no specific grant from any funding agency in the public, commercial, or not-for-profit sectors.

\section{Author Contributions}

Conceptualization - C.J.S., M.K., P.O., and A.A. Data acquisition/curation - C.J.S. and M.K. Data analysis/interpretation - C.J.S., M.M., and M.K. Writing - original draft - C.J.S., A.K.M., and M.M. Writing - reviewing and editing - C.J.S., A.K.M., M.M., M.K., P.O., P.B., and A.A.

\section{References}

1 Schrock AB, Ouyang C, Sandhu J, Sokol E, Jin D, Ross JS, et al. Tumor mutational burden is predictive of response to immune checkpoint inhibitors in MSI-high metastatic colorectal cancer. Ann Oncol. 2019 Jul 1;30(7):1096-103.

2 Fabrizio DA, George TJ Jr, Dunne RF, Frampton G, Sun J, Gowen K, et al. Beyond microsatellite testing: assessment of tumor mutational burden identifies subsets of colorectal cancer who may respond to immune checkpoint inhibition. J Gastrointest Oncol. 2018 Aug;9(4):610-7.

3 Goodman AM, Sokol ES, Frampton GM, Lippman SM, Kurzrock R. Microsatellite-stable tumors with high mutational burden benefit from immunotherapy. Cancer Immunol Res. 2019 0ct;7(10):1570-3.

4 Fakih MG, Padmanabhan A. CEA monitoring in colorectal cancer. What you should know. Oncology. 2006; 20(6):579.

5 Yan C, Hu Y, Zhang B, Mu L, Huang K, Zhao H, et al. The CEA/lo colorectal cancer cell population harbors cancer stem cells and metastatic cells. Oncotarget. 2016 Dec 6;7(49):80700-15.

6 Reinert T, Henriksen TV, Christensen E, Sharma S, Salari R, Sethi H, et al. Analysis of plasma cell-free DNA by ultradeep sequencing in patients with stages i to iii colorectal cancer. JAMA Oncol. 2019 May 9;5(8):1124-31.

7 Chen EX, Jonker DJ, Loree JM, Kennecke HF, Berry SR, Couture F, et al. Effect of combined immune checkpoint inhibition vs best supportive care alone in patients with advanced colorectal cancer: the canadian cancer trials group C0.26 study. JAMA Oncol. 2020;6(6):831-8.

8 Parikh AR, He Y, Hong TS, Corcoran RB, Clark JW, Ryan DP, et al. Analysis of DNA damage response gene alterations and tumor mutational burden across 17,486 tubular gastrointestinal carcinomas: implications for therapy. Oncologist. 2019 Oct;24(10):1340-7.

9 Parikh AR, Mojtahed A, Schneider JL, Kanter K, Van Seventer EE, Fetter IJ, et al. Serial ctDNA monitoring to predict response to systemic therapy in metastatic gastrointestinal cancers. Clin Cancer Res. 2020 Apr 15; 26(8):1877-85.

10 Cabel L, Riva F, Servois V, Livartowski A, Daniel C, Rampanou A, et al. Circulating tumor DNA changes for early monitoring of anti-PD1 immunotherapy: a proof-of-concept study. Ann Oncol. 2017 Aug 1;28(8):1996-2001. 